
allemande

45-2 | 2013

Images et discours de la nation

\title{
Le détachement international de travailleurs en droit allemand, autrichien et suisse
}

Chronique juridique

\section{Sandie Calme}

\section{OpenEdition}

\section{Journals}

Édition électronique

URL : https://journals.openedition.org/allemagne/1784

DOI : 10.4000/allemagne.1784

ISSN : 2605-7913

\section{Éditeur}

Société d'études allemandes

Édition imprimée

Date de publication : 30 décembre 2013

Pagination : 483-488

ISSN : 0035-0974

\section{Référence électronique}

Sandie Calme, "Le détachement international de travailleurs en droit allemand, autrichien et suisse », Revue d'Allemagne et des pays de langue allemande [En ligne], 45-2 | 2013, mis en ligne le 29 juillet 2019 consulté le 28 juin 2022. URL : http://journals.openedition.org/allemagne/1784 ; DOI : https://doi.org/ 10.4000/allemagne.1784 


\section{Chronique juridique \\ Le détachement international de travailleurs en droit allemand, autrichien et suisse}

\section{- Sandie Calme*}

La directive n 96/71/CE du Parlement et du Conseil du 16 décembre 1996 concernant le détachement de travailleurs effectué dans le cadre d'une prestation de services concerne les situations de détachement de travailleurs intracommunautaires, c'està-dire (article $1^{\text {er }}$ ) soit le détachement, par une entreprise intracommunautaire, pour son compte et sous sa direction, sur le territoire d'un État membre, dans le cadre d'un contrat conclu entre l'entreprise d'envoi et le destinataire de la prestation de services opérant dans cet État membre, pour autant qu'il existe une relation de travail entre l'entreprise d'envoi et le travailleur pendant la période de détachement (article $1^{\text {er }}, 3 .$, a)), soit le détachement d'un travailleur sur le territoire d'un autre État membre, dans un établissement ou dans une entreprise appartenant au groupe, pour autant qu'il existe une relation de travail entre l'entreprise d'envoi et le travailleur pendant la période de détachement (article $1^{\text {er }}, 3$., b)), soit le détachement, en tant qu'entreprise de travail intérimaire ou en tant qu'entreprise qui met un travailleur à disposition, d'un travailleur à une entreprise utilisatrice établie ou exerçant son activité sur le territoire d'un État membre, pour autant qu'il existe une relation de travail entre l'entreprise de travail intérimaire ou l'entreprise qui met un travailleur à disposition et le travailleur pendant la période de détachement (article $1^{\text {er }}, 3$., c)). Toutefois, le personnel navigant de la marine marchande est exclu de ce dispositif (article $1^{\text {er }}$, 2.) et la directive reconnaît le principe selon lequel les entreprises sises dans un État non membre ne peuvent bénéficier d'un traitement plus favorable que celui des entreprises intracommunautaire (article ${ }^{\text {er }}, 4$.). La directive institue un socle minimum de prérogatives impératives pour les salariés détachés quant à leurs conditions de travail, elle impose aux États membres de coopérer entre eux et de communiquer ouvertement sur leurs conditions nationales d'emploi,

* Docteur en droit, LL.M. (Francfort sur le Main, Allemagne), avocate au barreau de Paris. 
en vue de parvenir à ses objectifs de régulation et de transparence des conditions de travail des travailleurs détachés dans un esprit de non-discrimination et afin de pallier le risque de distorsion de la concurrence par une inégalité de traitement des travailleurs détachés qui consisterait à les exploiter abusivement à moindre coût. Une proposition de directive du Parlement européen et du Conseil relative à l'exécution de la directive 96/71/CE concernant le détachement de travailleurs effectué dans le cadre d'une prestation de services est à l'étude. La proposition s'inscrit dans le prolongement de la directive et vise à en assurer l'exécution en apportant des précisions. Elle aspire à ce que les sanctions soient pleinement efficaces, proportionnées et dissuasives et puissent être mutuellement reconnues et exécutées. Nous verrons que ce projet doit nourrir une réflexion allant au-delà du cadre intracommunautaire, en prenant l'exemple de l'Allemagne, de l'Autriche et de la Suisse.

\section{La mise en œuvre du droit de l'Union européenne sur le détachement international de travailleurs}

Pour ce qui est de la mise en œuvre du droit de l'Union européenne, le droit allemand et le droit autrichien ont élaboré leur système de protection des travailleurs détachés à l'international en suivant la directive mais en empruntant des voies différentes. Le droit allemand met l'accent sur l'alignement sur la protection offerte au niveau national alors que le droit autrichien opère une distinction selon que l'employeur qui détache le salarié siège ou non au sein de l'Espace Économique Européen ou en Suisse.

\section{A. En droit allemand}

En droit allemand, le détachement de travailleurs relève de la loi sur les conditions de travail impératives pour les employés et employées détachés à l’international et pour ceux et celles régulièrement engagés sur le territoire allemand (Gesetz über zwingende Arbeitsbedingungen für grenzüberschreitend entsandte und für regelmäßig im Inland beschäftigte Arbeitnehmer und Arbeitnehmerinnen), dite loi sur le détachement (Arbeitnehmer Entsendegesetz - AEntG). Cette loi a également vocation à assurer une loyale concurrence en matière de détachement $(\$ 1)$. Le détachement des travailleurs intéressant particulièrement l'actualité et la réalité du marché du travail, cette loi du 20 avril 2009 a succédé à celle du 26 février 1996, qu'elle a abrogée. Ainsi, la protection accordée aux travailleurs détachés est alignée sur celle de travailleurs non détachés régulièrement employés sur le territoire allemand. Ces conditions de travail impératives relèvent de conventions collectives, qui peuvent émaner notamment du ministère allemand du Travail. La protection se rapporte au salaire, aux congés, à la protection sociale en matière de santé et de travail et à la mise à disposition de main-d'œuvre: selon l'article $\$ 2$ de cette loi, les conditions de travail impératives portent sur la rémunération minimale y compris quant aux heures supplémentaires, sur les congés payés, les durées maximales de travail, les durées minimales de congés, les conditions du transfert de main-d'œuvre, y compris en matière de travail temporaire, la sécurité, la santé et l'hygiène au travail, les mesures de protection concernant les conditions de travail et d'emploi des femmes enceintes et des femmes après la grossesse, des enfants et des jeunes, l'égalité de traitement entre hommes et femmes et la lutte contre les discriminations ( $\$ 2)$. Aussi, les conditions impératives de travail prônées par cette loi portent sur des domaines d'activités circonscrits qui présentent une certaine diversité et vont au-delà du cadre 
des prestations de services relatifs à la construction. Le concept de salaire minimum relève de conventions collectives par secteur d'activité. Des dispositions spéciales portent une attention particulière à des branches d'activités telles que le domaine sanitaire $(\$ 10$ à $\$ 13)$, où une commission spéciale prend en charge l'élaboration des conditions de travail minimales, le transport routier de monnaie européenne ( $\$ 14)$.

En outre, l'entrepreneur ayant recours à un autre entrepreneur pour des prestations de services ou pour la réalisation d'un ouvrage répond des obligations de son soustraitant, de l'entrepreneur qu'il se substitue ultérieurement ou du prêteur mandaté par l'entrepreneur ou par l'entrepreneur lui succédant, en ce qui concerne le paiement aux employés de la rémunération minimale ou le versement de prestations sociales à un établissement commun aux parties à la convention collective et se rapportant aux conditions de travail impératives ( $\$ 14)$.

Le détachement des travailleurs fait l'objet d'une déclaration et d'un contrôle administratifs qui donnent lieu à une coopération internationale avec les administrations étrangères. Les sanctions peuvent consister en le versement d'une amende (\$23) voire en l’exclusion de marchés publics $(\$ 21)$.

\section{B. En droit autrichien}

D’après l'article $\$ 7$ de la loi d'adaptation du droit des contrats de travail (Arbeitsvertragsrechtsanpassungsgesetz), si un employeur non sis en Autriche et non membre d'une collectivité émettrice de conventions collectives en Autriche emploie un salarié dont le lieu de travail habituel se situe en Autriche, alors ce salarié a impérativement droit, au minimum, à la rémunération prévue par la loi, les règlements et accords collectifs, qui revient de droit aux salariés se trouvant dans sa situation sur son lieu de travail, pour des employeurs comparables.

Selon l'article $₫ 7 \mathrm{a}$ alinéa $1^{\text {er }}$ de la loi d'adaptation du droit des contrats de travail, la protection offerte par l'article $\$ 7$ s'étend, quel que soit le droit applicable à la relation de travail, à tout salarié détaché en Autriche par un employeur non sis dans un État membre de l'Espace Économique Européen pour des travaux s'effectuant dans le cadre d'un transfert de main-d'œuvre ou pour une prestation de travail suivie. L'employeur et l'entreprise cliente de ce dernier répondent solidairement de la rémunération minimale du travailleur ( $\$ 7$ a alinéa 2$)$. Les prérogatives du salarié portent notamment sur les congés et sur le temps de travail (article $\$ 7$ a alinéa 3). Pour des prestations de courte durée en matière de travaux d'assemblage et de réparations en lien avec la livraison d'équipement et de machines à une entreprise ou pour les travaux nécessaires à la mise en service de tels équipements et machines qui ne peuvent pas être réalisées par des salariés locaux, il est fait exception à ces prérogatives pour les salariés (article $\$ 7 \mathrm{a}$ alinéa 4 ). Par contre, pour les salariés employés pour des travaux de construction servant à ériger, mettre ou maintenir en état, reconstruire ou démolir des travaux de construction, les prérogatives en matière de congés, de temps de travail et de rémunération minimale sont de droit à compter du premier jour de travail en Autriche (article $\$ 7$ a alinéa 5).

Larticle $\$ 7 \mathrm{~b}$ de la loi d'adaptation du droit des contrats de travail se rapporte aux situations où l'employeur étranger a son siège social au sein d'un État de l'Espace Économique Européen et aux cas dans lesquels les salariés sont détachés en Autriche par un employeur sis en Suisse, pour une prestation de travail continue (article $7 \mathrm{~b}$ alinéa 10). Il accorde aux salariés détachés la même protection qu'à ceux dont l'employeur se situe 
en dehors de l'Espace Économique Européen et de la Suisse. À cela s'ajoute le respect de la directive $n^{\circ}$ 91/533/CEE du Conseil, du 14 octobre 1991, relative à l'obligation de l'employeur d'informer le travailleur des conditions applicables au contrat ou à la relation de travail. Le détachement des travailleurs par un employeur sis au sein de l'Espace Économique Européen ou en Suisse fait l'objet d'un contrôle administratif et d'une déclaration obligatoires, les documents spécifiques devant être conservés. Les contrôles font l'objet d'une coopération internationale entre les administrations compétentes. En particulier, toute violation des obligations de déclaration, tout manquement au formalisme inhérent à la relation de travail, sont susceptibles de donner lieu au versement d'une amende.

\section{II.Le positionnement du droit suisse}

La conceptualisation juridique suisse de la libre circulation des travailleurs appelle naturellement la Confédération helvétique à accorder sa règlementation sur le détachement des travailleurs avec la progression de cette liberté.

\section{A. Le positionnement du droit suisse en matière de libre circulation des travailleurs}

De par sa situation géographique, la Suisse est appelée à entretenir des relations économiques avec ses voisins de l'Union européenne, notamment en matière de relations de travail et, partant, de détachement de travailleurs. C'est pourquoi elle a entrepris un rapprochement de sa législation d'avec le droit intracommunautaire, érigé à certains égards en modèle. Ainsi, l'article 7, a), de l'accord entre la Confédération suisse, d'une part, et la Communauté européenne et ses États membres, d'autre part, sur la libre circulation des personnes, prône « le droit à l'égalité de traitement avec les nationaux en ce qui concerne l'accès à une activité économique et son exercice ainsi que les conditions de vie, d'emploi et de travail ». Cet accord a institué un Comité mixte composé de représentants des parties à l'accord, qui veille activement à l'ajustement de la législation suisse au droit communautaire, pour ce qui est de la réalisation des objectifs de libre circulation. Il est relayé par l'ordonnance sur l'introduction progressive de la libre circulation des personnes entre, d'une part, la Confédération suisse et, d'autre part, l'Union européenne et ses États membres, ainsi qu'entre les États membres de l'Association européenne de libre-échange, dite ordonnance sur l'introduction de la libre circulation des personnes. Cette évolution juridique progressive de la libre circulation des personnes va de pair avec le développement du droit suisse des détachements internationaux de travailleurs.

\section{B. L'ouverture du droit suisse en matière de détachement international de travailleurs}

En droit suisse, la régulation du détachement international de travailleurs relève de la loi fédérale sur les mesures d'accompagnement applicables aux travailleurs détachés et aux contrôles des salaires minimaux prévus par les contrats-types de travail, dite loi sur les travailleurs détachés. Elle porte, notamment en réponse aux travaux de Gysin Hans Rudolf dans le cadre de ses activités parlementaires jusqu'en décembre 2011, un intérêt particulier à la situation des faux indépendants, les travailleurs indépendants travaillant en Suisse faisant l'objet d'un contrôle administratif et étant soumis à l'obligation de procéder à des formalités spécifiques. Le traitement des salariés détachés est aligné sur celui prévu par les dispositions obligatoires en matière de rémunération 
minimale, de durée du travail, des vacances et du repos, de sécurité, de santé et d'hygiène au travail, de protection des femmes enceintes et des accouchées, des enfants et des jeunes, de non-discrimination et d'égalité de traitement entre hommes et femmes (article 2, alinéa $1^{\mathrm{er}}$ ), pendant toute la durée de la mission (article 2, alinéa 4).

Les dispositions des conventions collectives obligatoires s'appliquent aux travailleurs détachés dans les domaines suivants:

- les contributions obligatoires aux frais de formation continue, pour un détachement en Suisse de plus de 90 jours;

- le dépôt par l'employeur d'une garantie financière;

- les amendes dues à des organes paritaires chargés de veiller à la bonne application de l'accord collectif en cas de violation des normes relatives aux conditions minimales de travail et de salaire des travailleurs détachés.

Spécifiquement concernant la situation des personnes détachées, les paiements effectués par l'employeur au titre du détachement sont inclus dans le salaire dans la mesure où ils ne correspondent pas au remboursement de dépenses liées au détachement, concernant par exemple les frais de séjour (article 2 alinéa 3). L'employeur peut être tenu de verser des contributions sociales en Suisse (article 2 alinéa 5). Il s'agit notamment de la participation aux frais de contrôle et de fonctionnement des organes paritaires relevant des conventions collectives de travail obligatoires, qu'ils incombent aux travailleurs ou aux employeurs (article 8a de l'ordonnance sur les travailleurs détachés en Suisse). L'hébergement offert par l'employeur aux travailleurs détachés en Suisse doit respecter les conditions d'hygiène et de dignité usuelles (article 3). Les garanties salariales offertes par des organismes suisses de protection sociale du fait de conventions collectives de travail obligatoires concernent les travailleurs détachés en Suisse, à moins que l'employeur sis à l'étranger ne démontre verser à une institution comparable du lieu de son siège social de telles contributions pour la période de détachement en Suisse (article 2 alinéa 2).

Pour des travaux de faible ampleur ou de courte durée, des dérogations sont apportées en matière de rémunération et de vacances, sauf pour certains secteurs tels que la construction, le génie civil, le second œuvre, l'hôtellerie ou la restauration (article 4).

Dans le cadre de la sous-traitance (article 5), l'entrepreneur principal doit se charger de conduire le sous-traitant à respecter le droit suisse, au moyen d'un contrat. En cas de défaillance de sa part, il est sujet à des sanctions et engage sa responsabilité civile, étant entendu qu'il est solidairement responsable avec le sous-traitant en cas de nonrespect des conditions minimales de travail et de rémunération (article 5).

En principe, le détachement des travailleurs donne lieu à une déclaration administrative, à un contrôle et à l'obligation d'émettre et de conserver certains documents. Les administrations suisses procèdent au contrôle de façon coordonnée et en coopération avec les organismes de l'État du siège de l'employeur. Dans ce cadre, des sanctions peuvent être prises à l'encontre de l'employeur: elles peuvent consister en des amendes, en une interdiction d'activité professionnelle en Suisse pour une durée d'un an à cinq ans ou en l'obligation d'assumer personnellement les frais afférents au contrôle. En outre, la violation des dispositions protectrices des travailleurs détachés, dont notamment l'obstruction aux contrôles administratifs, peut conduire à des poursuites pénales (article 12). 
À l'instar de la proposition de directive intracommunautaire, le législateur suisse a eu l'occasion de se préoccuper des modalités d'exécution de ses sanctions administratives quant aux violations des normes sur les travailleurs détachés, notamment concernant un jugement par lequel un tribunal allemand (Arbeitsgericht Ulm, Urteil vom 29. Juli 2009 Az. 2 Ca 571/08), en tant que juge (compétent conformément à la convention de Lugano) de l'entreprise débitrice sise en Allemagne ayant procédé à la violation du droit suisse sur le détachement international de travailleurs, a appliqué le droit allemand quant au paiement des sanctions administratives, de manière à anéantir ces sanctions. Ces difficultés ont donné lieu à des discussions au sein d'un groupe de travail trinational d'experts entre l'Allemagne, l'Autriche et la Suisse. La proposition de directive présente donc un intérêt majeur de réflexion pour le droit suisse afin d'harmoniser les normes relatives au détachement international de travailleurs. S'il est vrai que la directive $n^{\circ}$ 96/71/CE prive les entreprises extracommunautaires de tout traitement plus favorable par rapport à celui des entreprises intracommunautaires, un alignement de fait du droit suisse sur le droit de l'Union européenne à l'image des transpositions nationales intracommunautaires de la directive est d'autant plus envisageable que le législateur suisse procède par une analyse approfondie de la teneur du droit intracommunautaire. 